\title{
SALUD SEXUAL EN MUJERES QUE TIENEN SEXO CON MUJERES: LAGUNAS Y DESAFÍOS PARA PROFESIONALES DE SALUD.
}

\author{
Cynthia Vergara-Maldonado \\ Instituto de Salud Sexual y Reproductiva \\ Universidad Austral de Chile \\ cynthia.vergara@uach.cl \\ Angel Gasch- Gallén \\ Facultad de Ciencias de la Salud \\ Universidad de Zaragoza. \\ angelgasch@unizar.es
}

\begin{abstract}
RESUMEN
Existen datos poco claros acerca de la frecuencia de las Infecciones de Transmisión Sexual (ITS) entre las mujeres que tienen sexo con mujeres (MSM) y se desconocen las intervenciones más eficaces para asegurar sus autocuidados en este campo. Por tanto, el objetivo de la investigación se centró en identificar los conocimientos, lagunas y recomendaciones existentes en la literatura científica en materia de salud sexual en las MSM, para lo cual se realizó una revisión tipo scoping sobre la salud sexual en MSM desde el año 2000 hasta el 2019. Se extrajo información sobre las características clave de los estudios y la calidad de la evidencia, mediante las categorías como: salud sexual integral, especificación de prácticas sexuales en MSM y recomendaciones de profesional en salud sexual y se excluyeron estudios con falta de enfoque en la salud sexual en MSM.

Se seleccionaron 39 estudios, en su mayoría transversales. Las lagunas identificadas se centran en la ausencia de evidencia sobre la salud sexual, la confusión sobre la orientación sexual y las prácticas sexuales, la falta de interés específico en la salud sexual integral y el escaso enfoque en el ciclo vital. Las recomendaciones se orientaron al autocuidado de las MSM, las intervenciones dirigidas a la práctica clínica, la investigación, la educación y la prevención de los profesionales de la salud aunque sin un enfoque a lo largo del curso de la vida. Por tanto, existen varias lagunas en materia de salud sexual en la MSM. La ausencia de datos publicados sobre los problemas de salud de las MSM perpetúa el modelo heteronormativo de atención sanitaria, situándolas en escenarios de desigualdad. Las evidencias existentes apuntan a la necesidad de intervenir desde la mejora del autocuidado con una mirada integral. Este es un aspecto de excelencia para los profesionales de salud para desarrollar estrategias.
\end{abstract}

Palabras clave: practicas sexuales; salud sexual; cuidados en salud; mujeres que tienen sexo con mujeres; lesbianas.

\begin{abstract}
The prevalence of sexually transmitted infections (STDs) among women who have sex with women (WSW) is unclear, and the most effective interventions to ensure their self-care in this area are unknown. Therefore, the aim of the research focused on identifying the existing knowledge, gaps and recommendations in the scientific literature on sexual health in WSW
\end{abstract}


by conducting a scoping review on sexual health in WSW from 2000 to 2019. Information was extracted on the key characteristics of the studies and the quality of the evidence, through categories such as: comprehensive sexual health, specification of sexual practices in WSW and recommendations of sexual health professionals, and studies with a lack of focus on sexual health in WSW were excluded.

Thirty-nine studies, mostly cross-sectional, were selected. Gaps identified centred on the absence of evidence on sexual health, confusion about sexual orientation and sexual practices, lack of specific interest in comprehensive sexual health and little focus on the life course. Recommendations were oriented towards WSW self-care, interventions aimed at clinical practice, research, education and prevention by health professionals but without a life-course approach. Thus, there are several gaps in sexual health in WSW. The absence of published data on the health problems of WSW perpetuates the heteronormative model of health care, placing them in unequal settings. Existing evidence points to the need to intervene by improving self-care with a holistic approach. This is an aspect of excellence for health professionals to develop strategies.

Keywords: sexual practices; sexual health; health care; wsw; lesbian. 


\section{INTRODUCCIÓN}

La atención sanitaria con enfoque integral que abarque toda la diversidad afectivo-sexual de las personas requiere una comprensión de aspectos muy específicos que expliquen la diversidad de realidades y que partan de la adquisición de competencias específicas por parte de los profesionales de salud para el abordaje integral de los cuidados y atención sanitaria. En nuestro contexto disponemos de pocos estudios que analicen las diversas prácticas sexuales y sus cuidados, así como las actitudes y competencias de las y los profesionales de salud hacia los cuidados y atención a personas que viven la diversidad afectivo-sexual y de género.

Uno de los grupos donde existen apenas estudios o datos epidemiológicos claros que exploren, por ejemplo, las ITS (infecciones de transmisión sexual), corresponde a la población de las mujeres que tienen sexo con mujeres (MSM) (Blondeel et al., 2016). Existen apenas estudios donde se analicen los comportamientos sexuales dentro de este colectivo (Doull et al., 2018). Incluso suelen demostrar que algunas enfermedades son más prevalentes entre las (MSM), más que en la población general o las mujeres heterosexuales (Zeeman et al., 2017). No obstante, otros demuestran que algunas de estas enfermedades son producto de los estilos de vida y de la estigmatización por orientación sexual (Gil-Borrelli et al., 2017). Por tanto, se hace difícil precisar estrategias desde los profesionales de salud, tanto a nivel de atención sanitaria como en el ámbito de salud comunitaria, en respuesta a las necesidades de las MSM.

Existen aproximaciones que nos señalan que a pesar de los enormes avances que se han realizado en materia de derechos civiles y aceptación social en el mundo para la población LGTBI, las MSM sufren una enorme discriminación en materia de salud, con miedo a revelar su orientación sexual e identidad sexual. La situación incluye la falta de profesionales informados acerca de sus necesidades y capaces de orientarlas correctamente (McKay, 2011).

Es preciso entender que muchas veces las MSM son tratadas como grupo homogéneo o de menor riesgo (Dolan \& Davis, 2003). La explicación radica en que el contacto de las mujeres heterosexuales con los servicios de salud es mayor, debido a la natalidad, uso de anticoncepción, entre otras (Polek \& Hardie, 2017). Esta situación hace que se conozca más sobre sus necesidades en salud, al contrario de las MSM, y solo se conoce de ellas porque algunas han tenido relaciones sexuales con hombres y acuden a los centros de salud.

Asimismo, los profesionales de salud parecen no tener claro las necesidades específicas de salud y según avanzaban en edad, declaran sentirse menos competentes para proveer cuidados a personas Lesbianas, Gais, Bisexuales, y Transexuales (LGBT), por tanto, demandaban más formación en el abordaje de las barreras a la expresión y vivencia de la diversidad afectivo-sexual. Por lo mismo, se recomienda una "competencia profesional de sexo-género en la práctica sanitaria asistencial" y crear indicadores para medir la magnitud de la existencia de dicha competencia en los centros asistenciales (Gasch \& Tomas, 2017).

Lo anterior, puede estar impactando en el cuidado de salud integral de un grupo específico del colectivo como son las MSM, más aún cuando los estudios parecen centrarse más en las poblaciones de hombres que tienen sexo con hombres (HSH) y VIH (Zaidi et al., 2016). Por ende, es necesario cuestionarse y reflexionar sobre cuáles son los desafíos para los profesionales de salud en cuanto a estrategias y cuidados frente a las necesidades en materia de salud sexual para dicha población. 
Por consiguiente, el objetivo de nuestra investigación radica en identificar los conocimientos, lagunas y recomendaciones existentes en la literatura científica en materia de salud sexual en las MSM, enfocándonos en la salud sexual integral los cuidados según prácticas sexuales, conjunto las estrategias y recomendaciones para los profesionales de salud en la atención sanitaria de esta población.

\section{METODOLOGÍA}

Se realizó una revisión integral de la evidencia científica sobre la salud sexual en MSM desde el año 2000 hasta el 2019 en Bases de Datos PubMed-Scielo-Lilacs-Embase-CINAHL. Se extrajo información mediante las categorías como: "salud sexual integral", "prácticas sexuales en MSM" y "recomendaciones para profesionales de salud", con sus homólogos en inglés arrojando un total de 3569 artículos. Se realizó la revisión exhaustiva a través de título y Abstract de cada artículo y tras la aplicación de los criterios de exclusión, quedaron finalmente un total de 39 artículos (ver Figura 1).

\section{Figura 1}

Flujograma de Estrategia Metodológica de Selección

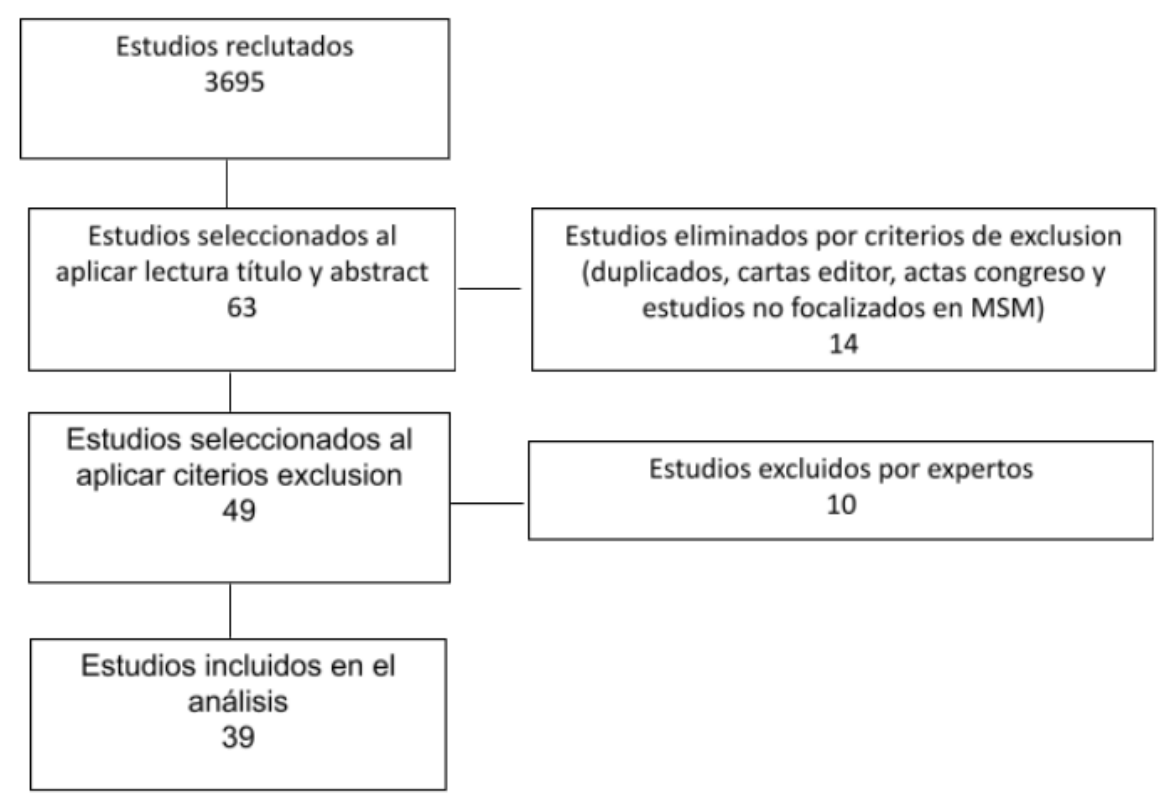

Para el análisis se crearon matrices con las categorías de búsqueda, marcando con técnica de fichaje los datos importantes para cada ítem y reordenando la base en una nube one drive con clave que solo manejaban investigadores del estudio. Esta investigación es parte de un proyecto internacional autogestionado "WSW Sexual health and STDs" de los autores, y cuenta con la aprobación del Comité de Ética de Aragón.

\section{RESULTADOS}


De los artículos que forman parte de estudio, se obtuvieron dispares tamaños poblaciones, variando entre 15 y 5714 mujeres, cuyas edades no sobrepasan los 55 años. La mayoría de los estudios provienen de Norte América y Europa. Se presentaron estudios focalizados en diversas ITS, entre ellos 23 estudios (59\%) se centraron en ITS específicas, y 17 estudios $(43 \%)$ en ITS desde la generalidad. No obstante, los datos presentados son poco claros al referirse a cada ITS, en función de prevalencia, mecanismo de contagio, y cuidados dirigidos hacia MSM. Tal es el caso de la vaginosis bacteriana (VB) cuya prevalencia en la MSM oscila entre $14 \%$ a $43 \%$ según estudios señalados (Forcey et al., 2015). Otros estudios se centraron en otras ITS, tales como Clamidia, Virus papiloma humano (VPH), Virus Herpes simple, entre otras (Eaton et al., 2008; Muzny et al., 2016; Xu et al., 2010), encontrándose hallazgos poco claros sobre la prevalencia por lo que coinciden los resultados. No obstante, llama la atención que no se encontraron estudios en cuanto a ITS y prácticas sexuales en mujeres mayores de edad, inclusive en ninguno de estos estudios se profundizó en el impacto tanto en la infancia como en la adolescencia, sobre todo en materias como el uso o no de barreras al tener relaciones sexuales (Doull et al., 2018; Muzny et al., 2013).

Entre los estudios, tampoco se hace referencia a los cuidados específicos orientados a las diversas prácticas sexuales, confundiéndose muchas veces con orientación sexual, y no se evidencian recomendaciones sobre tipos de estrategias para abordar la prevención y promoción, tanto en el ámbito comunitario como de atención sanitaria propiamente tal. Dicho de otro modo, las recomendaciones, aunque destinadas al autocuidado de las MSM y a guiar profesionales de salud con campañas o educación, no describen formas de ejecutar dichos cuidados de manera específica o realizar las estrategias a nivel comunitario, con una clara ausencia de un enfoque multidisciplinario a la hora de abordar a la población MSM.

Asimismo, sorprende que la mitad de los estudios señalan abordar la salud sexual desde un punto de vista integral, no obstante, su enfoque se orienta desde la patología y no involucran al placer como parte de la salud sexual de las MSM.

\section{DISCUSIÓN}

Las lagunas identificadas en los estudios dan cuenta de la generalización en el abordaje de la atención sanitaria, centrándose mayormente en el autocuidado y la higiene, que en estrategias de los equipos de salud para orientar cuidados desde el comportamiento y prácticas sexuales en MSM. Incluso, dichas aproximaciones a la salud sexual de las MSM, no han considerado la diversidad de mujeres y sus interseccionalidades, cuestión que ya es sugerida por otro estudio (Cox \& McNair, 2009). Tampoco es considerado el riesgo por práctica sexual determinada sino más bien se confunde con un riesgo basado en la orientación sexual, cuestión que aún no deja claro los enfoques para actuar como profesionales de salud.

Los estudios igualmente coinciden en que las MSM usan pocas barreras de protección al contacto sexual con otras mujeres, esto puede explicar por las dificultades de acceso y diagnóstico en atención hacia esta población, descrita en otro estudio, donde se demuestra incluso que dichas barreras en la atención repercuten en las MSM que igualmente no perciben riesgo en sus prácticas sexuales (Silberman et al., 2016). 
Existe una ausencia, en los estudios encontrados, de los cuidados en salud sexual de MSM en el curso de vida, esto puede deberse a que las poblaciones estudiadas de MSM no sobrepasan los 55 años, por tanto, epidemiológicamente es posible que no tengamos el conocimiento suficiente acerca de la prevalencia de ITS específicamente en esta población de edad mayor o bien no acuden a los centros de salud. Lo anterior, demuestra que se debe abordar una mirada sanitaria capaz de relacionar los cuidados en las practicas sexuales, con las condicionantes sociales y el género estudiadas en poblaciones LGTBI, más aún en la vejez, ya que en este curso de vida las mujeres pertenecientes a este colectivo vivencian un nuevo renacer sexo-afectivo en edades mayores (Schultze, 2019). Por consecuencia, lo anterior nos desafía a futuras investigaciones y abordajes acerca de los cuidados en salud sexual, el placer y otros aspectos relacionados con las vivencias de la sexualidad en MSM de edades mayores.

En los estudios seleccionados se señala que la salud sexual de las MSM es vista desde un punto de vista integral. No obstante, queda un espacio que profundizar en cuanto a las intervenciones en ITS así como en otros aspectos ligados a la salud sexual desde la promoción y prevención tanto a nivel local como comunitario para abordar integralmente dicho aspecto.

El abordaje de la salud sexual en los estudios da cuenta de un enfoque centrado en lo curativo, por lo que incluso desde la promoción y prevención en salud sexual, se queda al debe la formación de profesionales de salud con contenidos que aborden la salud sexual en su diversidad, cuestión que coincide con otros estudios (Vergara \& Gasch, 2019), por ejemplo, donde se describe la existencia de aproximaciones que detallan un avance legislativo que propicia a la apertura hacia el tema evidenciando que a pesar de ello en materia de salud sexual en relación a la población MSM se ha avanzado poco, por tanto, solo existen intentos en abordar la diversidad en la formación de profesiones de salud como matronas y enfermeras de manera incipiente. Dicho de otro modo, esto constituye una oportunidad para incorporar a la formación de todos los profesionales de salud en el currículum.

Asimismo, los estudios recomiendan realizar estrategias para profesionales de salud, tanto a nivel de atención sanitaria como de intervención en sus centros de salud, estos debiesen implicar promoción y prevención, con campañas destinadas a la población de MSM así como a la población general según práctica sexual inclusive y si es preciso utilizando las plataformas de internet para la educación sexual (Vergara, 2018). Además, los estudios encontrados señalan que se debe fortalecer la promoción de la investigación en la materia y el diseño de pautas específicas para la atención sanitaria de MSM. Sin duda un desafío para estudios futuros en relación a la ejecución de dichas estrategias a nivel multidisciplinar.

Finalmente, en este estudio, se lograron identificar las lagunas y desafíos para los profesionales de salud, los que nos indican posibles intervenciones futuras y nuevas investigaciones, combinando métodos cualitativos y cuantitativos, cuestión que puede ser una limitante de nuestro estudio, al solo centrarnos en una revisión panorámica. Otra limitación puede ser el escaso catastro de publicaciones enfocadas a la salud de las MSM solamente y que se encuentren en revistas de impacto, por lo que se recomienda ampliar la búsqueda a otras revistas y documentos dado la poca información que existe.

\section{CONCLUSIONES}


La salud sexual de las MSM presenta lagunas en cuanto al abordaje integral de la salud sexual en el curso de vida. Las conclusiones de este estudio se pueden extrapolar al panorama general que existe sobre la MSM y nos sugiere seguir investigando y desafiándonos como equipos de salud desde un punto de vista multidisciplinario.

Se hace necesario entender la salud sexual integral a través del curso de vida, incorporando el placer y la educación sexual para todos los grupos de mujeres considerando sus interseccionalidades tanto en sus dimensiones psicológicas, sociales y físicas, sobre todo en poblaciones de edad adulta mayor MSM.

Los datos epidemiológicos poco claros acerca de las ITS nos desafían a realizar estudios con poblaciones de MSM en diversos territorios geográficos y culturas, contribuyendo en alianzas cooperativas con profesionales de salud que se desempeñan lugares con escasas investigaciones o avances legislativos y de salud como Latinoamérica y otros.

Para el desarrollo de las recomendaciones de este estudio y la ejecución de estrategias en salud sexual, es menester convocar a profesionales de diversas disciplinas (psicología, kinesiología, matronería, enfermería, medicina, ciencias políticas, antropología, etc.). Por lo que, en la actualidad, se hace necesario el estudio de futuros planes a nivel comunitario y sanitario constituyéndose un desafío inclusive para quienes estar a cargo de formar los futuros profesionales y para otras(os) profesionales relacionados con la salud que realizan investigación o gestión en salud, incorporando estrategias en sus lugares de trabajo. Por tanto, si bien las recomendaciones apuntan al autocuidado, parece existir un vacío en relación a recomendar acciones a nivel gubernamental que impliquen cambios en los programas de salud apuntando a la estructura del sistema sanitario.

Todo lo anterior, perpetua el modelo heteronormativo en la atención sanitaria y comunitaria, por lo que las lagunas vislumbradas en la salud sexual de las MSM pueden otorgar las pistas necesarias a nivel sanitario para evolucionar en los modelos de atención hacia modelos más integrales en salud sexual.

\section{REFERENCIAS}

Blondeel, K., Say, L., Chou, D., Toskin, I., Khosla, R., Scolaro, E., y Temmerman, M. (2016). Evidence and knowledge gaps on the disease burden in sexual and gender minorities: A review of systematic reviews. International Journal for Equity in Health, 15(1), 1-9. https://doi.org/10.1186/s12939-016-0304-1

Cox, P., y McNair, R. (2009). Risk reduction as an accepted framework for safer-sex promotion among women who have sex with women. Sexual Health, 6(1), 15-18. https://doi.org/10.1071/SH08022

Dolan, K. A., y Davis, P. W. (2003). Nuances and shifts in lesbian women's constructions of STI and HIV vulnerability. Social Science and Medicine, 57(1), 25-38. https://doi.org/10.1016/S0277-9536(02)00305-2

Doull, M., Wolowic, J., Saewyc, E., Rosario, M., Prescott, T., y Ybarra, M. L. (2018). Why Girls Choose Not to Use Barriers to Prevent Sexually Transmitted Infection During Female-to-Female Sex. Journal of Adolescent Health, 62(4), 411-416. 
https://doi.org/10.1016/j.jadohealth.2017.10.005

Eaton, L., Kalichman, S., Cain, D., Cherry, C., Pope, H., Fuhrel, A., y Kaufman, M. (2008). Perceived prevalence and risks for human papillomavirus (HPV) infection among women who have sex with women. Journal of Women's Health, 17(1), 75-83. https://doi.org/10.1089/jwh.2006.0256

Forcey, D. S., Vodstrcil, L. A., Hocking, J. S., Fairley, C. K., Law, M., McNair, R. P., y Bradshaw, C. S. (2015). Factors associated with bacterial vaginosis among women who have sex with women: A systematic review. PLOS ONE, 10(12), 1-16. https://doi.org/10.1371/journal.pone.0141905

Gasch, A., y Tomas, C. (2017, septiembre 6-8). Las carencias en el enfoque de género en la práctica asistencial: una llamada a la acción y análisis preliminar. Congreso de epidemiología, XII Congresso da associação, Barcelona, España.

Gasch, A., y Tomas, C. (2017). Las carencias en el enfoque de género en la práctica asistencial: una llamada a la acción y análisis preliminar. Gaceta Sanitaria, 31, 152. Recuperado de: https://www.reunionanualsee.org/2017/documentos/Gaceta Sanitaria.pdf

Gil-Borrelli, C. C., Velasco, C., Iniesta, C., de Beltrán, P., Curto, J., y Latasa, P. (2017). Hacia una salud pública con orgullo: equidad en la salud en las personas lesbianas, gais, bisexuales y trans en España. Gaceta Sanitaria, 31(3), 175-177. https://doi.org/10.1016/j.gaceta.2016.09.013

McKay, B. (2011). Lesbian, Gay, Bisexual, and Transgender Health Issues, Disparities, and Information Resources. Medical Reference Services Quarterly, 30(4), 393-401. https://doi.org/10.1080/02763869.2011.608971

Muzny, C. A., Harbison, H. S., Pembleton, E. S., Hook, E. W., y Austin, E. L. (2013). Misperceptions regarding protective barrier method use for safer sex among African-American women who have sex with women. Sexual Health, 10(2), 138-141. https://doi.org/10.1071/SH12106

Muzny, C. A., Kapil, R., Austin, E. L., Brown, L. D., Hook, E. W., y Geisler, W. M. (2016). Chlamydia trachomatis infection in African American women who exclusively have sex with women. International Journal of STD and AIDS, 27(11), 978-983. https://doi.org/10.1177/0956462415604092

Polek, C., y Hardie, T. (2017). Changing HPV vaccination rates in bisexual and lesbian women. Journal of the American Association of Nurse Practitioners, 29(6), 333-339. https://doi.org/10.1002/2327-6924.12453

Schultze, F. R. (2019). Diversidad sexual y envejecimiento, los cursos de vida de la poblacion LGTB Argentina. Revista Iluminurias, 20(49), 230-261. https://doi.org/10.22456/1984-1191.93297

Silberman, P., Buedo, P. E., y Burgos, L. M. (2016). Barreras en la atención de la salud sexual en Argentina: Percepción de las mujeres que tienen sexo con mujeres. Revista de Salud Pública, 18(1), 1-12. https://doi.org/10.15446/rsap.v18n1.48047 
Vergara, C. (2018). Miradas de los grupos LGBT en Chile para diseñar un programa de educación sexual a través de la red. Revista Nemesis, 14, 65-87. Recuperado de http://revistanemesis.cl/wp-content/uploads/2018/08/Miradas-de-los-grupos-LGBT-en-C hile.pdf

Vergara, C., y Gasch, A. (2019). Evolución de salud de las mujeres lesbianas en chile percepción de la atención sanitaria 2007-2018. Gaceta Sanitaria, 33, 178-179. Recuperado de https://www.gacetasanitaria.org/es-pdf-X0213911119000670

Xu, F., Sternberg, M. R., y Markowitz, L. E. (2010). Women who have sex with women in the United States: Prevalence, sexual behavior and prevalence of herpes simplex virus type 2 infection-Results from national health and nutrition examination survey 2001-2006. Sexually Transmitted Diseases, 37(7), 407-413. https://doi.org/10.1097/OLQ.0b013e3181db2e18

Zaidi, S. S., Ocholla, A. M., Otieno, R. A., y Sandfort, T. G. M. (2016). Women Who Have Sex with Women in Kenya and Their Sexual and Reproductive Health. LGBT Health, 3(2), 139-145. https://doi.org/10.1089/lgbt.2014.0121

Zeeman, L., Sherriff, N., Browne, K., McGlynn, N., Aujean, S., Pinto, N., Davis, R., Mirandola, M., Gios, L., Amaddeo, F., Donisi, V., Rosinska, M., Niedźwiedzka-Stadnik, M., y Pierson, A. (2017). Reducing health inequalities felt by LGBTI people.

Recuperado de:

https://ec.europa.eu/health/sites/default/files/social determinants/docs/2018 lgbti modu $\underline{\text { le2 en.pdf }}$ 